examinations, and the like; when the heart is examined during some minor illness, often a respiratory infection; or when a child is examined to discover why growth and development are abnormal. In the first two situations the parents are worried, anxious, and hoping that nothing has been found to indicate that the heart is diseased. In the third the parents are usually no less worried and anxious, but they are often hoping that something has been found which, if treated, may allow normal growth. Nowadays, however, few deleterious congenital malformations of the heart escape early detection. Murmurs discovered by chance are seldom loud, nearly always systolic, and usually limited to early and mid-systole. They are accompanied by normal heart sounds that vary normally with the respiratory cycle. They do not radiate to any of the classical sites and may vary considerably with posture and respiration. They often have a musical, grating, or vibratory quality and sound superficial, almost as though they were between the heart and the stethoscope rather than inside the heart and great vessels. Perhaps most important of all, they do not suggest a diagnosis. Various methods have been used to grade their intensity, but because with training the ear registers sounds that become progressively louder and more obvious to the listener, intensity for practical purposes depends largely on experience and what one is accustomed to hearing. Thus a family doctor expecting and accustomed to silence during systole, may be concerned about a murmur that would seem of relatively little account to a cardiologist.

The use of the adjectives "inorganic" and "functional", so often applied to murmurs heard in the absence of apparent heart disease, has done little to clarify a situation that obviously puzzles many of those who have to make decisions about their meaning. Clearly something must be causing a murmur, and these epithets have little relevance to its possible aetiology. Sometimes the cause is obvious: anaemia, venous hums, fevers, cardio-respiratory movements, and bony abnormalities of the thoracic cage are a few. Often it is not, and no amount of detailed investigation will do other than exclude obvious causes. Recent studies on haemodynamic aspects of left ventricular function in children have shed new light on a possible cause of some by showing that up to the age of 15 years the pre-ejection time appears to be shorter than in adults. ${ }^{2}$ In children with vibratory praecordial murmurs it is shorter than in normal children, ${ }^{3}$ which suggests that some murmurs may be attributed to a higher contractility of the myocardium resulting in a higher peak flow through the aortic ostium. A positive correlation has been found between pubertal development and the disappearance of murmurs, and also that pre-ejection times return to normal in children whose murmurs have disappeared. ${ }^{4}$

Many murmurs are caused by minor malformations. Stenoses in the main branches of the pulmonary arteries and tiny septal defects are common examples. These are innocent lesions, and the term "innocent," by which is meant that it has no haemodynamic significance and will affect neither the way of life nor the expectation of life of the person in whom it is heard, is the best way to describe all unimportant murmurs. The term also has the advantage that it begs the question of aetiology, which is of no consequence once a decision that it is an innocent noise has been made.

The detection of a heart murmur is always a major happening in a child's life and all too often initiates a chain of events that may profoundly influence a lifetime. Much unnecessary worry and invalidism result in cases in which it would have been far better if the doctor had never listened or at least had never said what he heard. On hearing a murmur it is therefore extremely important to decide what to take seriously and what to ignore. The seeds of a cardiac neurosis can be planted while the patient is still very young, and if once allowed to germinate they are difficult to eradicate. As the vast majority of murmurs donotsignify heart disease, the medical examiner must be careful not to give the impression that the heart may be abnormal. If he is satisfied that the child is healthy, the murmur will almost certainly disappear as the child grows up, and he should keep his counsel. But if he suspects an abnormality and, being uncertain of its significance, relates his findings to the patient or the parents the child should be referred straight away for an expert opinion, so that doubts and fears have no chance to develop.

\footnotetext{
1 Lessof, M., and Brigden, W., Lancet, 1957, 2, 673.
van der Hoeven, G. M. A., de Monchy, C., and Beneken, J. E. W., British Heart fournal, 1973, 35, 669 .

${ }^{3}$ de Monchy, C., van der Hoeven, G. M. A., and Beneken, J. E. W., British Heart fournal, 1973, 35, 679.

4 de Monchy, C., van der Hoeven, G. M. A., and Beneken, J. E. W., British Heart fournal, 1973, 35, 685.
}

\section{Resuscitation for Students}

Groups of doctors in Britain who have organized themselves to provide effective care as soon as possible after road accidents now include about 800 of the total of 23,000 general practitioners. It is not possible at present to measure their effectiveness in preventing deaths: estimates ${ }^{1-4}$ of lives that could be saved have varied from $20-25 \%$ of those dying after road accidents to less than $10 \%$, but the only definite British figures support the smaller estimates. ${ }^{5}$ Remarkably enough, the doctors concerned in these schemes have trained and equipped themselves for the purpose and have been supported by voluntary subscription.

The many uncertainties in this field recently prompted an inquiry into the instruction of medical students on major trauma and resuscitation, ${ }^{6}$ and the resulting report gives information from 74 medical schools in 22 countries in different parts of the world. The time devoted to the theory and practice of resuscitation varies widely, but it is not clear just what students are taught or what they practise. E. Hoffman suggests that "emergency medical care should be included in the curriculum as an independent multidepartmental topic" and that instruction should start in the second preclinical year with a one to two weeks' optional course that could include travelling in ambulances. In the first clinical year there would be a further one to two weeks' training with another two months in the second year and a nine weeks' elective period in the third. However much goodwill there may be towards improving the standard of immediate care of the victims of injury and sudden illness, it could hardly be expected to extend to setting aside as much of the students' time as that. One wonders also how easy it would be to persuade pathologists, physiologists, anaesthetists, cardiologists, and general and special surgeons to construct and provide the necessary courses. To ask these questions is not to dismiss the suggestions so much as to inquire whether they could be met and, particularly, without overburdening the curriculum. Successful management of major trauma and cardiopulmonary resuscitation calls in the first place for prompt recognition that a threat to life exists and for the means of mitigating it; this may give way directly to a period of intensive care to deal with continuing threats to life, and the newly qualified doctor should have at least basic competence in these subjects. He has less need for competence in the definitive treatment of 
major illness or major injury because this allows him to turn to others in a way that an emergency does not.

Knowledge of the pathological and physiological features of the potentially fatal effects of illness and injury will in any case be provided by cardiologists, anaesthetists, and others, and if there is some repetition this is not a bad thing. Practical experience is needed as well. Attendance in receiving rooms and resuscitation and other intensive care units should be compulsory-and it should be arranged so as to ensure that the students will see and do what is necessary. Ideally they should be given a measure of responsibility under supervision, and they should be there when the work needs to be done. This means weekend and night duty, particularly in the accident department. A busy week in an intensive care unit and another in an accident and emergency receiving room towards the end of his clinical training should enable the newly qualified doctor to acquit himself at least reasonably well in the emergencies that he has to deal with. Travelling in ambulances is a good way of learning what ambulance crews can and do do, and a day based on a busy emergency station would probably suffice, but it offers little opportunity for resuscitation.

One may question, then, the need for at least two to three months' instruction and training in emergency care as a separate subject; but the necessary theoretical knowledge must be imparted to students and they should have adequate exposure to the rigours of emergency care, for which some will have to go beyond their teaching hospitals.

1 Läuppi, E., Schweizerische medinizische Wochenschrift, 1954, 84, 335.

2 Mackay, G. M., British Medical fournal, 1969, 4, 799.

3 Ruffell Smith, H. P., Injury, 1970, 2, 99.

4 London, P. S., Injury, 1971/72, 3, 225.

5 Snook, R., British Medical fournal, 1972, 3, 569.

6 Snook, R., British Medical fournal, 1972,

\section{Implications of Optic Neuritis}

When treating patients with optic neuritis it would be a great help if it were possible to identify those likely to develop multiple sclerosis. After exclusion of possible causes such as diabetes mellitus, vitamin $B_{12}$ deficiency, Leber's optic atrophy, toxins including methanol, tobacco, and many other drugs, postinfective encephalomyelitis, and ischaemic lesions of the optic nerve, ${ }^{12}$ a substantial number of patients remain in whom no certain diagnosis can be made. Somewhere between $11.5 \%$ and $85 \%$ of these patients later develop definite multiple sclerosis ${ }^{3}{ }^{4}$-the generally accepted figure $e^{5}$ is about $30 \%$. Multiple sclerosis may still be responsible in the remainder, and such patients can never be considered entirely free of risk, since the longest interval between the attack of optic neuritis and the development of signs and symptoms of definite multiple sclerosis has been 37 years. $^{6}$

Patients with multiple sclerosis who present with optic neuritis often seem to have a milder form of the disease, though this is not agreed by all. ${ }^{7}$ In trying to identify the patients who will develop multiple sclerosis the clinical details of the optic neuritis are of no help. ${ }^{5}$ So far there is no specific test for multiple sclerosis of proved accuracy. The test suggested by J. Mertin, B. K. Shenton, and E. J. Field ${ }^{8}{ }^{9}$ has been advanced as specific, but their results have yet to be substantiated on a larger number of patients.
Several other abnormalities have been reported by workers investigating large groups of patients with multiple sclerosis. Some of the studies referred to in a recent paper by H. Link, E. Norrby, and J.-E. Olsson ${ }^{10}$ showed a high proportion of such patients with raised titres of measles antibodies in the cerebrospinal fluid compared with controls, a lowered ratio between the serum titre and that in the cerebrospinal fluid, and the presence of oligoclonal IgG in the cerebrospinal fluid. Using these tests, Link and his colleagues in the virological department of the Karolinska Institute, Stockholm, examined 41 patients with optic neuritis who were less than 50 years of age at presentation, who had had no previous neurological incidents, and in whom there were no signs of other neurological disease. They found approximately half had oligoclonal IgG in the cerebrospinal fluid (group 1), while the other half did not (group 2). There was some suggestion of clinical differences between the groups, with pain on movement of the eye, severe depression of vision, a progressive course over three months, and optic disc abnormalities being common in group 1, while heavier consumption of alcohol and tobacco were commoner in group 2. Several other differences were also found. More patients in group 1 showed pleocytosis and an increase in the total protein and percentage of IgG in the cerebrospinal fluid; and these patients also had a pronounced increase in haemolysis-inhibiting measles virus antibody titres in their cerebrospinal fluid. The proportion of patients with a reduced ratio of the serum to cerebrospinal fluid measles antibody titres was also markedly increased in group 1. The implications of these abnormalities in measles antibodies and IgG in multiple sclerosis are still uncertain, though it has been suggested that in those patients with increased measles antibodies measles virus (or some agent with identical antigenic features) is the slow virus against which the body's immunological system is reacting to produce the disease multiple sclerosis.

It is important to await the results of follow-up before concluding that patients in group 1 of Link and colleagues are more likely to develop multiple sclerosis, but the data do suggest a process with similar antigenic features to multiple sclerosis is occurring in these patients and that more of the central nervous system is affected than just the optic nerves, even though there are no clinical signs and symptoms of such wider extent of the disease.

The Swedish paper was accompanied by a leading article by B. G. W. Arnason ${ }^{11}$ drawing attention to work showing that histocompatibility types HL-A 3 and HL-A 7 are overrepresented in those with multiple sclerosis, and that this preponderance is absent in patients presenting with optic neuritis but who have no evidence of multiple sclerosis over a period of several years. The genetic determinant designated LD-7a is apparently found in $70 \%$ of patients with multiple sclerosis and only $10 \%$ of controls, indicating that carriers of this determinant have a 15 times increased risk of developing multiple sclerosis.

Other reports of familial predisposition to multiple sclerosis have recently appeared both in scientific publications and in the lay news media. J. H. McIntyre ${ }^{12}$ reported four cases of multiple sclerosis with a family history of the disease. Field and colleagues ${ }^{13}$ found evidence of abnormality in the macrophage migration inhibition test in $41 \%$ of relatives of patients with multiple sclerosis, particularly female relatives. As a result of the widespread dissemination of the latter preliminary report in the lay news media unnecessary concern has been caused to relatives of patients with multiple sclerosis. The results of a great many studies have indicated ${ }^{14}$ that the risks to siblings and children of patients with multiple sclerosis 